University of Windsor

Scholarship at UWindsor

$9-2013$

\title{
A NOVEL APPROACH TO CHARACTERIZING HESITATIONS IN INTUITIONISTIC FUZZY NUMBERS
}

Minji Huang

Xiamen University

Kevin Li

University of Windsor

Follow this and additional works at: https://scholar.uwindsor.ca/odettepub

Part of the Business Commons

\section{Recommended Citation}

Huang, Minji and Li, Kevin. (2013). A NOVEL APPROACH TO CHARACTERIZING HESITATIONS IN INTUITIONISTIC FUZZY NUMBERS. Journal of Systems Science and Systems Engineering, 22 (3), 283-294.

https://scholar.uwindsor.ca/odettepub/94

This Article is brought to you for free and open access by the Odette School of Business at Scholarship at UWindsor. It has been accepted for inclusion in Odette School of Business Publications by an authorized administrator of Scholarship at UWindsor. For more information, please contact scholarship@uwindsor.ca. 


\title{
A NOVEL APPROACH TO CHARACTERIZING HESITATIONS IN INTUITIONISTIC FUZZY NUMBERS*
}

\author{
Minji Huang ${ }^{1}$ Kevin W. Li ${ }^{2}$ \\ ${ }^{1}$ Department of Automation, Xiamen University, Xiamen Fujian 361005, China \\ Huangmj@xmu.edu.cn \\ ${ }^{2}$ Odette School of Business, University of Windsor, Windsor Ontario N9B 3P4, Canada \\ kwli@uwindsor.cn(ه)
}

\begin{abstract}
Building upon the concept of $D_{\alpha}$ operator introduced by Atanassov (1989), this article proposes an improved objective approach and a hybrid approach to operationalize $D_{\alpha}$ so that the hesitation in an intuitionistic fuzzy number (IFN) can be further refined and characterized. Numerical experiments are carried out to demonstrate the features of the proposed approach and its novelty compared to existing methods in the literature. The aim is to furnish an effective way to refine hesitations in intuitionistic fuzzy assessments for more reliable and confident decision aids.
\end{abstract}

Keywords: Intuitionistic fuzzy numbers, fuzzy numbers, hesitation, $D_{\alpha}$ operator

\section{Introduction}

Since Atanassov (1986) introduced intuitionistic fuzzy sets (IFSs), IFSs have been widely recognized as an important generalization of fuzzy sets (FSs) (Zadeh 1965). In addition to a membership function to express the degree of an element's belongingness to a particular fuzzy set, an IFS includes a nonmembership function to reflect the degree of an element's nonbelongingness to the set. Any remaining information that is not captured by these two functions in an IFS indicates the DM's hesitation or indeterminacy in the assessment, often referred to as the intuitionistic fuzzy index
(IFI) of an IFS.

Due to their flexibility in characterizing uncertainty in human cognitive processes, IFSs have been widely applied to group and multicriteria decision making problems arising in a wide range of fields (Atanassova 2006, Boran and Genc et al. 2009, Chen and Wang et al. 2011, Herrera and Marttínez et al. 2005, Tan and Chen 2010, Wang and Li 2012, Wang and Li et al. 2011, Xu 2011, Xu and Chen 2011). In these decision approaches, an inevitable procedure is how to rank intuitionistic fuzzy numbers (IFNs) for making a final choice after assessments on decision alternatives are

\footnotetext{
* The research is financially supported by the funding (No.JA09291) from the Fujian Education Department, China, the Natural Sciences and Engineering Research Council of Canada (NSERC) under its Discovery Grant program and the Natural Science Foundation of China (Nos. 71272129 and 71271188).
} 
aggregated into IFNs.

Although different ranking methods have been put forward, the score function introduced by Chen and Tan (1994) stands out as the most widely used basis for comparing IFNs. Their score function is defined as the difference between the membership and nonmembership function (See Definition 2.4) and, hence, does not account for hesitations inherent in IFN assessments. As pointed out by Chen (2011), other forms of score functions are also devised to handle multicriteria decision making under different environments.

Subsequently, an accuracy function (Hong and Choi 2000), defined as the sum of the membership and nonmembership functions (See Definition 2.5), is proposed to gauge the complement of the hesitation, but the ranking method typically takes a prioritized order (Wang and $\mathrm{Li}$ et al. 2009, Li and Wang 2010). As such, if two IFNs can be differentiated by their score function values, the accuracy function is usually not entertained. In this case, the hesitation will be effectively excluded from the ranking process This treatment may sometimes create problems, especially when an IFN contains a large hesitation, signifying the DM's high uncertainty or risk level. Under such a condition, a simple discarding of hesitations in IFNs may yield risky or misleading recommendations. For instance, given two IFNs $A=[0.1,0]$ and $B=[0.5,0.5]$, the comparison result is $A>B$ based on the score function approach (Chen and Tan, 1994). From a voting perspective, IFN $A$ can be roughly interpreted as 1 out of 10 "approval", no "opposition", and 9 out of 10 "abstentions"; IFN $B$ can be loosely treated as 5 out of 10 "for" votes, 5 out of 10 "against" votes, and no "abstentions". It is clear that $A$ contains a significant amount of hesitations but $B$ contains no hesitation at all. To obtain a more accurate ranking between these two IFNs, it is sensible to further characterize the $90 \%$ hesitations in $A$ rather than completely discard it.

One way to handle hesitations in IFNs is the so-called $D_{\alpha}$ operator proposed by Atanassov (1989, 1995, 2008). The essence of this operator is to split the hesitation into two parts, with one part being added to the membership function and the remaining part being attributed to the nonmembership function. This treatment basically reduces an IFN to a fuzzy number (FN) and the amount of hesitations that is attributed to the membership or nonmembership function depends on a parameter $\alpha$. To further characterize hesitations, Liu and Wang (2007) extend Atanassov's $D_{\alpha}$ operator and furnish a dual-parameter operator $F_{\alpha, \beta}$,where $0 \leq \alpha, \beta \leq 1$ and $\alpha+\beta \leq 1$, and $\alpha$ determines how much of the hesitation is attributed to the membership function and $\beta$ establishes how much of the hesitation is ascribed to the nonmembership function. It is apparent that $F_{\alpha, \beta}$ is reduced to $D_{\alpha}$ if $\alpha+\beta=1$. As such, Liu and Wang (2007)'s research includes Atanassov's $D_{\alpha}$ operator as a special case. In contrast to Atanassov's $D_{\alpha}$ operator that transforms an IFN to an FN, Liu and Wang (2007)'s approach converts an IFN with a larger IFI into an IFN with a smaller IFI after their operator is applied for a finite number of times. In addition, Liu and Wang (2007) prove that the limit of their dual-parameter operator is equivalent to a specific $D_{\alpha}$ operator.

While Atanassov $(1989,1995,2008)$ and Liu and Wang (2007) have introduced the $D_{\alpha}$ and 
$F_{\alpha, \beta}$ operators and investigate their generic properties, no specific formulas are furnished to determine the values of $\alpha$ and/or $\beta$. This deficiency makes it hard for an analyst to apply these operators in decision problems involving IFN assessments with large hesitations. To address this issue, Wang and Lei et al. (2007) proposed different approaches to characterize the $D_{\alpha}$ operator. After examining these existing methods and pointing out their technical deficiencies, this article puts forward a novel hybrid approach to refine the hesitation in an IFN.

The rest of the article is organized as follows, Section 2 furnishes the basics of IFSs and the associated $D_{\alpha}$ operator and Section 3 reviews existing methods in implementing this operator. Section 4 proposes an improved formula for $\alpha$ and carries out comparative studies between existing and the proposed method. Section 5 describes a hybrid approach to operationalizing $D_{\alpha}$ and the paper concludes with some comments in Section 6.

\section{Preliminaries}

In this section, some basic concepts of FSs and IFSs and the associated operator $D_{\alpha}$ are introduced to facilitate future discussions.

Definition 2.1 Let $X$ be a fixed set of universe, a fuzzy set $F$ in $X$ is defined as a set of ordered pairs (Zadeh 1965):

$$
F=\left\{\left\langle x, \mu_{F}(x)\right\rangle \mid x \in X\right\}
$$

where $\mu_{F}(x): X \rightarrow[0,1]$ is a membership function, denoting the degree of membership of element $x \in X$ to $F$.

Atanassov (1986) introduced an extension of fuzzy sets called Intuitionistic Fuzzy Sets (IFSs) as follows:
Definition 2.2 Let $X$ be a fixed set of universe, an IFS $F$ in $X$ is defined as:

$$
F=\left\{\left\langle x, \mu_{F}(x), v_{F}(x)\right\rangle \mid x \in X\right\}
$$

where $\mu_{F}(x), v_{F}(x): X \rightarrow[0,1] \quad$ characterize the degree of membership and nonmembership of element $x \in X$ to $F$, respectively, and for $\forall x \in X, 0 \leq \mu_{F}(x)+v_{F}(x) \leq 1$

$$
\pi_{F}(x)=1-\mu_{F}(x)-v_{F}(x)
$$

is usually called the intuitionistic fuzzy index (IFI), expressing the degree of hesitation or indeterminacy. It is obvious that $\pi_{F}(x) \in[0,1]$ for $x \in X$.

If $\forall x \in X, \pi_{F}(x)=0$, then $F$ is reduced to a normal fuzzy set.

To further characterize hesitations in an IFS, Atanassov (1989, 1995, 2008) defined an operator $D_{\alpha}$ as follows:

Definition 2.3 Let $\alpha \in[0,1]$ be a fixed number. For an IFS $F$, the operator $D_{\alpha}$ is defined as:

$$
D_{\alpha}(F)=\left\{<x, \mu_{F}(x)+\alpha \cdot \pi_{F}(x),-\mu_{F}(x)-\alpha \cdot \pi_{F}(x) \ngtr x \in \mathrm{X}\right\}
$$

(4) can be equivalently expressed as:

$$
D_{\alpha}(F)=\left\{<x, \mu_{F}(x)+\alpha \cdot \pi_{F}(x), v_{F}(x)+(1-\alpha) \cdot \pi_{F}(x) \ngtr \mid x \in \mathrm{X}\right\}
$$

As

$$
\begin{aligned}
& \mu_{F}(x)+\alpha \cdot \pi_{\mathrm{F}}(x)+v_{F}(x)+(1-\alpha) \cdot \pi_{\mathrm{F}}(x) \\
& =\mu_{F}(x)+v_{F}(x)+\pi_{F}(x)=1
\end{aligned}
$$

it is apparent that $D_{\alpha}$ effectively reduces an IFS $F$ to a fuzzy set with a membership function $\mu_{F}(x)+\alpha \cdot \pi_{\mathrm{F}}(x)$.

The nature of this $D_{\alpha}$ operator is to divide the IFI into two parts, and attribute part of the IFI to the membership function and the remainder to the nonmembership function. $\alpha$ serves as a key parameter to determine how much of the hesitation will be attributed to the membership and nonmembership functions, respectively.

For a particular element $x$, the pair $\left\langle\mu_{F}(x), v_{F}(x)\right\rangle$ is often referred to as an intuitionistic fuzzy number (IFN) (Xu and Yager 
2008, Chen and Yang 2012). Without causing confusion, an IFN can be simply denoted as $\beta=\langle\mu, v\rangle$, where, $\mu \in[0,1] \quad v \in[0,1]$ and $\mu+v \leq 1$. Without loss of generality, the following discussions are confined to IFNs and fuzzy numbers (FNs).

To compare two IFNs, Chen and Tan (1994) introduced a score function as follows:

Definition 2.4 For an IFN $\beta=\langle\mu, v\rangle$, its score function is defined as

$$
S_{\beta}=\mu-v
$$

It is apparent that $S_{\beta} \in[-1,1]$, and a larger score corresponds to a bigger IFN. Subsequently, Hong and Choi (2000) introduced an accuracy function to complement the score function in comparing IFNs.

Definition 2.5 For an IFN $\beta=\langle\mu, v\rangle$, its accuracy function is defined as

$$
H_{\beta}=\mu+v
$$

If an IFN is examined in the context of a voting process, the membership $\mu$ and nonmembership $v$ can be loosely interpreted as the percentage of "support" and "opposition" votes, respectively, and its IFI $\pi=1-\mu-v$ can be naturally regarded as the percentage of "neutrality" or "abstention" votes. Understandably, the higher an IFI in an IFN, the more uncertain or indeterminate the DM is about its assessment. Therefore, when an IFI is large, significant risk is imbedded in the decision process and it is worthwhile to further refine the IFI and elicit the DM's tendency towards membership or nonmembership in its assessment.

The $D_{\alpha}$ operator furnishes a generic mathematical framework for further refining IFI in an IFN, and $\alpha$ specifies the percentage of the hesitation to be attributed to the membership
( $\alpha$ )and nonmembership ( $1-\alpha)$ functions, thereby reducing an IFN to an FN. Then, it is natural to question how $\alpha$ should be determined. In the next section, we shall review existing approaches to characterizing $\alpha$, followed by our proposed method in Section 4.

\section{Existing Approaches to Determining $\alpha$}

Wang and Lei et al. (2007) propose several specific approaches to determine $\alpha$, including the so-called average, proportion, and difference-adjustment method as detailed below:

\subsection{The Average Method}

For an IFN $F=\left\langle\mu_{F}, v_{F}\right\rangle$, let

$$
\alpha=\frac{1}{2}
$$

then $F$ can be reduced to an FN $\tilde{F}$ with a degree of membership:

$$
\mu_{\tilde{F}}=\mu_{F}+\frac{1}{2} \pi_{F}
$$

where $\pi=1-\mu_{F}-v_{F}$ is the IFI of $F$.

It is obvious that this average method simply splits the hesitation into two halves and adds each half to the membership and nonmembership degree, respectively. It is our opinion that this treatment is too simplistic.

\subsection{The Proportion Method}

$$
\text { For an IFN } \begin{aligned}
F & =\left\langle\mu_{F}, v_{F}\right\rangle, \text { let } \\
\alpha & =\frac{\mu_{F}}{\mu_{F}+v_{F}}
\end{aligned}
$$

then $F$ can be reduced to an FN $\widetilde{F}$ with a degree of membership:

$$
\mu_{\widetilde{F}}=\mu_{F}+\frac{\mu_{F}}{\mu_{F}+v_{F}} \pi_{F}
$$

In the proportion method, the percentage of 
the hesitation attributed to the membership (nonmembership) degree is proportional to the known membership (nonmembership) level as given in $\mu_{F}\left(v_{F}\right)$. This treatment, to a certain degree, reflects the "following-the-herd" principle in characterizing the hesitation: if the known membership (nonmembership) takes a higher proportion in the accuracy function as defined in Definition 2.5, a larger percentage of the hesitation will be added to the membership (nonmembership) degree in the conversion process. We think that this is a more sensible way to characterize the hesitation in an IFN.

It is worth noting that the only formulas to determining the values of $\alpha$ and $\beta$ are provided in Theorem 3.1 (iii) in Liu and Wang (2007), where $\alpha=\frac{\mu_{F}}{\mu_{F}+v_{F}}$ and $\beta=\frac{v_{F}}{\mu_{F}+v_{F}}$. It is obvious that $\beta=1-\alpha$ and $F_{\alpha, \beta}$ is reduced to $D_{\alpha}$. Therefore, these two formulas are equivalent to the proportion method put forward by Wang and Lei et al. (2007) and reviewed herein.

Based on this method, if the known membership (nonmembership) approaches zero, little or no hesitation will be attributed to the membership (nonmembership) degree. This property may not be desirable.

\subsection{The Difference-adjustment Method}

For an IFN $F=\left\langle\mu_{F}, v_{F}\right\rangle$, let

$$
\alpha=0.5+\frac{\mu_{F}-v_{F}}{2}
$$

then $F$ can be reduced to an $\mathrm{FN} \widetilde{F}$ with a degree of membership:

$$
\mu_{\widetilde{F}}=\mu_{F}+\left(0.5+\frac{\mu_{F}-v_{F}}{2}\right) \cdot \pi_{F}
$$

The $\alpha$ value here, to a certain degree, considers both the aforesaid average and proportion methods. It takes 0.5 as the base point and is adjusted by one half of the difference between $\mu_{F}$ and $v_{F}$. Therefore, for a given nonmembership (membership) degree in an IFN, the higher the known membership (nonmembership) degree is, the more hesitation is attributed to the membership (nonmembership) in the induced FN. Due to the base point 0.5 , this treatment usually yields a positive attribution to the membership (nonmembership) degree even if the known membership (nonmembership) is zero.

The formula for $\alpha$ in (13) can be rewritten as follows:

$$
\alpha=\frac{1+\mu_{F}-v_{F}}{2}=\frac{2 \mu_{F}+\pi_{F}}{2}=\mu_{F}+\frac{\pi_{F}}{2}
$$

Eq. (15) clearly indicates that the percentage of hesitations attributed to the membership function equals the membership plus one half of the IFI. Therefore, as long as there exists a positive indeterminacy in the IFN, the attribution of hesitations to the membership degree will always be positive in the conversion process. Apparently, if there does not exist any indeterminacy in the IFN assessment, it becomes irrelevant to discuss the attribution of hesitations.

Given the score function in Definition 2.4 (Chen and Tan 1994, Chen 2011), from (13), one can see that the attribution of hesitations to the membership (nonmembership) stays constant regardless the hesitation level as long as the IFN's score function remains the same. Next, an improved formula for $\alpha$ is proposed to refine the hesitation.

\section{An Improved Formula for $\alpha$} We propose the following formula to 
characterize $D_{\alpha}$.

$$
\alpha=\frac{1}{2}+\frac{\mu_{F}-v_{F}}{2}+\frac{\mu_{F}-v_{F}}{2} \cdot \pi_{F}
$$

Compared to (13) as given by Wang and Lei et al. (2007), this proposed formula has an additional term $\frac{\mu_{F}-v_{F}}{2} \cdot \pi_{F}$. This modification allows the $\alpha$ value to vary with not only the score function but also the IFI. It is apparent from (16) that $\alpha$ increases in $\pi_{F}$ for a given positive score function $S_{F}=\mu_{F}-v_{F}$ and decreases in $\pi_{F}$ for a given negative score function.

Equivalently, (16) can be expressed as:

$$
\alpha=\mu_{F}+\frac{\pi_{F}}{2}+\frac{S_{F}}{2} \cdot \pi_{F}
$$

As $S_{F} \in[-1,1] \quad, \quad$ it follows that $\mu_{F} \leq \alpha \leq \mu_{F}+\pi_{F} \leq 1$.

As the average method always evenly splits the IFI with $\alpha=\frac{1}{2}$, it is trivial for any further discussion. The following comparison will be focused on the remaining three methods. For conciseness, let $\alpha_{1}, \alpha_{2}, \alpha_{3}$ be the $\alpha$ value derived from the proportion, difference-adjustment, and our method, respectively. Then, the following properties hold true:

1) If $\mu_{F}=v_{F} \neq 0$, then $\alpha_{1}=\alpha_{2}=\alpha_{3}=0.5$;

2) If $\mu_{F}+v_{F}=1$, then $\alpha_{1}=\alpha_{2}=\alpha_{3}=\mu_{F}$;

3) If $\mu_{F} \leq v_{F}$, then $\alpha_{1} \leq \alpha_{3} \leq \alpha_{2}$;

4) If $\mu_{F} \geq v_{F}$, then $\alpha_{2} \leq \alpha_{3} \leq \alpha_{1}$.

Proof. The proof of 1) and 2) is trivial. To prove $3)$ note that the additional term $\frac{\mu_{F}-v_{F}}{2} \cdot \pi_{F} \leq 0$ given that $\mu_{F} \leq v_{F}$, hence, $\alpha_{3} \leq \alpha_{2}$. In addition,

$$
\begin{aligned}
& \alpha_{3}-\alpha_{1}=0.5+\frac{\mu_{F}-v_{F}}{2}+\frac{\mu_{F}-v_{F}}{2} \cdot \pi_{F}-\frac{\mu_{F}}{\mu_{F}+v_{F}} \\
& =\frac{\left[1+\mu_{F}-v_{F}+\left(\mu_{F}-v_{F}\right) \pi_{F}\right]\left(\mu_{F}+v_{F}\right)-2 \mu_{F}}{2\left(\mu_{F}+v_{F}\right)} \\
& =\frac{\left[1+\left(\mu_{F}-v_{F}\right)\left(1+\pi_{F}\right)\right]\left(\mu_{F}+v_{F}\right)-2 \mu_{F}}{2\left(\mu_{F}+v_{F}\right)} \\
& =\frac{-\left(\mu_{F}-v_{F}\right)+\left(\mu_{F}-v_{F}\right)\left(\mu_{F}+v_{F}\right)\left(1+\pi_{F}\right)}{2\left(\mu_{F}+v_{F}\right)} \\
& =\frac{\left(\mu_{F}-v_{F}\right)\left[\left(\mu_{F}+v_{F}\right)\left(1+\pi_{F}\right)-1\right]}{2\left(\mu_{F}+v_{F}\right)} \\
& =\frac{\left(\mu_{F}-v_{F}\right)\left[\left(1-\pi_{F}\right)\left(1+\pi_{F}\right)-1\right]}{2\left(\mu_{F}+v_{F}\right)} \\
& =\frac{-\pi_{F}^{2}\left(\mu_{F}-v_{F}\right)}{2\left(\mu_{F}+v_{F}\right)}
\end{aligned}
$$

Table 1A comparative study of four methods for determining the value of $\alpha$

\begin{tabular}{ccccc}
\hline IFN & Average & Proportion & $\begin{array}{c}\text { Difference- } \\
\text { adjustment }\end{array}$ & Our Method \\
\hline$<0,0>$ & 0.5 & N/A & 0.5 & 0.5 \\
\hline$<0,0.4>$ & 0.5 & 0 & 0.3 & 0.18 \\
\hline$<0.4,0>$ & 0.5 & 1 & 0.7 & 0.82 \\
\hline$<0.1,0.1>$ & 0.5 & 0.5 & 0.5 & 0.5 \\
\hline$<0.6,0.1>$ & 0.5 & 0.857 & 0.75 & 0.825 \\
\hline$<0.1,0.6>$ & 0.5 & 0.143 & 0.25 & 0.175 \\
\hline$<0.3,0.7>$ & 0.5 & 0.3 & 0.3 & 0.3 \\
\hline$<0.7,0.3>$ & 0.5 & 0.7 & 0.7 & 0.7 \\
\hline
\end{tabular}

As $\mu_{F} \leq v_{F}$, we have $\alpha_{3}-\alpha_{1} \geq 0$ $\Rightarrow \alpha_{3} \geq \alpha_{1}$. Property 3 ) is thus proved. Property 4) can be proved in a similar fashion.
Next, we shall conduct comparative studies with the different methods mentioned in Section 3. First, a comparison is carried out for the three 
Huang and Li: A novel approach to characterizing hesitations in intuitionistic fuzzy numbers

methods and our proposed formula for several IFNs and the result is displayed in Table 1.

Table 1 confirms that all the aforesaid approaches yield the same result $\alpha=0.5$ if $\mu_{F}=v_{F} \neq 0$. This result is reasonable given that the known membership and nonmembership are evenly distributed and an even split of the hesitation is thus sensible. Table 1 further verifies that, if $\mu_{F}+v_{F}=1$, corresponding to the case that there does not exist any hesitation in the IFN judgment, the last three approaches return the same value $\alpha=\mu_{F}$
(See the last two rows in Table 1). Of course, it is irrelevant here as there will be no hesitation to be characterized in this case. Table 1 also certifies that $\alpha_{1} \leq \alpha_{3} \leq \alpha_{2}$ if $\mu_{F} \leq v_{F}$ and $\alpha_{2} \leq \alpha_{3} \leq \alpha_{1}$ whenever $\mu_{F} \geq v_{F}$. Properties 3 ) and 4) indicate that the proposed method takes a more balanced approach between the proportion and the difference-adjustment method in refining the hesitation in an IFN assessment.

Table 2 A comparative study for the proportion, difference-amendment, and our method

\begin{tabular}{|c|c|c|c|c|c|}
\hline IFN & $S_{F}$ & $\pi_{F}$ & $\alpha_{1}$ & $\alpha_{2}$ & $\alpha_{3}$ \\
\hline$<0,0.8>$ & \multirow{3}{*}{-0.8} & 0.2 & 0 & 0.1 & 0.02 \\
\hline$<0.05,0.85>$ & & 0.1 & 0.056 & 0.1 & 0.06 \\
\hline$<0.1,0.9>$ & & 0 & 0.1 & 0.1 & 0.1 \\
\hline$<0,0.6>$ & \multirow{3}{*}{-0.6} & 0.4 & 0 & 0.2 & 0.08 \\
\hline$<0.1,0.7>$ & & 0.2 & 0.125 & 0.2 & 0.14 \\
\hline$<0.2,0.8>$ & & 0 & 0.2 & 0.2 & 0.2 \\
\hline$<0.1,0.5>$ & \multirow{3}{*}{-0.4} & 0.4 & 0.167 & 0.3 & 0.22 \\
\hline$<0.2,0.6>$ & & 0.2 & 0.25 & 0.3 & 0.26 \\
\hline$<0.3,0.7>$ & & 0 & 0.3 & 0.3 & 0.3 \\
\hline$<0.1,0.3>$ & \multirow{3}{*}{-0.2} & 0.6 & 0.25 & 0.4 & 0.34 \\
\hline$<0.2,0.4>$ & & 0.4 & 0.333 & 0.4 & 0.36 \\
\hline$<0.3,0.5>$ & & 0.2 & 0.375 & 0.4 & 0.38 \\
\hline$<0.1,0.1>$ & \multirow{3}{*}{0} & 0.8 & 0.5 & 0.5 & 0.5 \\
\hline$<0.2,0.2>$ & & 0.6 & 0.5 & 0.5 & 0.5 \\
\hline$<0.3,0.3>$ & & 0.4 & 0.5 & 0.5 & 0.5 \\
\hline$<0.2,0>$ & \multirow{3}{*}{0.2} & 0.8 & 1 & 0.6 & 0.68 \\
\hline$<0.3,0.1>$ & & 0.6 & 0.75 & 0.6 & 0.66 \\
\hline$<0.4,0.2>$ & & 0.4 & 0.667 & 0.6 & 0.64 \\
\hline$<0.4,0>$ & \multirow{3}{*}{0.4} & 0.6 & 1 & 0.7 & 0.82 \\
\hline$<0.5,0.1>$ & & 0.4 & 0.833 & 0.7 & 0.78 \\
\hline$<0.6,0.2>$ & & 0.2 & 0.75 & 0.7 & 0.74 \\
\hline$<0.6,0>$ & \multirow{3}{*}{0.6} & 0.4 & 1 & 0.8 & 0.92 \\
\hline$<0.7,0.1>$ & & 0.2 & 0.875 & 0.8 & 0.86 \\
\hline$<0.8,0.2>$ & & 0 & 0.8 & 0.8 & 0.8 \\
\hline$<0.8,0>$ & \multirow{3}{*}{0.8} & 0.2 & 1 & 0.9 & 0.98 \\
\hline$<0.85,0.05>$ & & 0.1 & 0.944 & 0.9 & 0.94 \\
\hline$<0.9,0.1>$ & & 0 & 0.9 & 0.9 & 0.9 \\
\hline
\end{tabular}


Next, a more detailed comparison among the last three methods is carried out to examine how the score function and IFI together affect the $\alpha$ value and the result is summarized in Table 2 .

Table 2 demonstrates that our proposed method yields an $\alpha$ value that is dependent upon both the score function and the hesitation level of an IFN. On the other hand, the difference-adjustment method produces an $\alpha$ value that is only contingent upon the score function but independent of the hesitation level. While the proportion method is able to take into account both the score function and IFI, our approach always gives a moderated $\alpha$ value that is bounded by those obtained from the other two methods.

Furthermore, this comparative study confirms that our method yields an $\alpha$ value decreasing in the IFI $\pi_{F}$ for a given negative score function $S_{F}$, but increasing in $\pi_{F}$ when $S_{F}$ is positive. This attribution rule, in our opinion, is reasonable in the sense of the "following-the-herd" principle: When the number of "support" vote exceeds that of "opposition" (corresponding to a positive score function), a larger percentage of the hesitation will be attributed to the membership function with a higher IFI (resulting in a larger $\alpha$ ); When "opposition" outnumbers "support" in a vote (implying a negative score function), more of the hesitation will be attributed to the nonmembership function with a larger IFI (corresponding to a smaller $\alpha$ ).

In summary, the proposed formula (16) yields an attribution rule that is consistent with the "following-the-herd" principle and takes both the score and hesitation functions into account. This improved approach is able to avoid extreme cases of attributing all hesitations to the membership function such as $\langle 0.2,0>$ in the proportion method $\left(\alpha_{1}=1\right)$. It also circumvents the problem that the attribution stays constant regardless of the hesitation level for any given score function $S_{F}$ in the difference-adjustment method.

\section{A Hybrid Approach to Determining}

$\alpha$

It should be recognized that the attribution of IFI in the conversion process actually depends on a DM's subjective judgment. Whenever possible, the DM who provides the initial IFN assessment should be consulted for further elicitation of its tendency towards membership and nonmembership in $\pi_{F}$. While the proposed method in Section 4 aims to provide an objective approach to determining $\alpha$, it is our opinion that the DM's subjective judgment should also be accommodated in order to obtain a more reliable attribution rule. This section puts forward a hybrid framework for determining $\alpha$. This hybrid approach is designed to integrate the DM's subjective judgment into an objective assessment.

If the DM is available for providing additional information to refine the hesitation reflected in the IFI, it is common that this is given as a set of linguistic variables (Wang and Qian 2007). These linguistic variables may be expressed as corresponding triangular fuzzy numbers (Li and Karray et al. 2001, Li 2002), which can then be converted to an appropriate $\alpha$ value. To facilitate the DM to furnish its subjective judgment for refining the IFI, the 
following table is proposed, which gives a range of graphic grids with corresponding linguistic labels. Seven levels are included in this conversion table and triangular fuzzy numbers are adopted to represent the linguistic variables (Wang and Qian 2007). It is apparent that a different number of levels and different fuzzifization and defuzzifization schemes may be applied as per the DM's preference.

The first column gives a corresponding graphic grid for the seven linguistic variables, where the bottom means a very large $\alpha$ value and the top indicates a very small $\alpha$. If this conversion table is adopted, the DM may use the graphic grid as a visual aid for specifying its judgment on $\alpha$. Alternatively, the DM may provide its judgment as an appropriate linguistic variable as given in Table 3 for conversion to a real value.

Table $3 \mathrm{~A}$ possible conversion grid for specifying $\alpha$

\begin{tabular}{cccc}
\hline \multirow{2}{*}{$\begin{array}{c}\text { Graphic } \\
\text { Grid }\end{array}$} & $\begin{array}{c}\text { Linguistic } \\
\text { Variable }\end{array}$ & $\begin{array}{c}\text { Triangular Fuzzy } \\
\text { Number }\end{array}$ & $\alpha$ \\
\hline \multirow{4}{*}{} & Very Small & $(0,0,0.2)$ & 0.050 \\
\cline { 2 - 4 } & Small & $(0,0.1,0.3)$ & 0.125 \\
\cline { 2 - 4 } & Slightly Small & $(0,0.2,0.4)$ & 0.200 \\
\cline { 2 - 4 } & Moderate & $(0.3,0.5,0.7)$ & 0.500 \\
\hline & Little Large & $(0.6,0.7,0.8)$ & 0.700 \\
\hline & Large & $(0.7,0.8,0.9)$ & 0.800 \\
\hline & Very Large & $(0.9,1.0,1.0)$ & 0.975 \\
\hline
\end{tabular}

It is apparent that Table 3 is purely based upon a DM's subjective judgment and the proposed method in Section 4 is, on the other hand, an objective approach to determining $\alpha$. In reality, a DM may wish to contain the subjectivity level to within a reasonable limit. In this case, a more desirable way is likely to be a hybrid framework that is able to integrate subjective judgment into an objective assessment. Based on this consideration, the following hybrid approach is put forward to specify the value of $\alpha$.

Let $\alpha^{s}$ be the value of $\alpha$ identified by the DM, $\alpha^{o}$ be the $\alpha$ value calculated by an objective method such as the one proposed in Section 4 , and $\varepsilon$ be a threshold parameter identified by the DM to represent an allowable range for the subjectivity, then the hybrid approach to refining the IFI is given by:

$$
\alpha=\left\{\begin{array}{ccc}
(1-\varepsilon) \alpha^{o} & \text { if } & \alpha^{s}<(1-\varepsilon) \alpha^{o} \\
\alpha^{s} & \text { if } & (1-\varepsilon) \alpha^{o} \leq \alpha^{s} \leq(1+\varepsilon) \alpha^{o} \\
(1+\varepsilon) \alpha^{o} & \text { if } & \alpha^{s}>(1+\varepsilon) \alpha^{o}
\end{array}\right\}
$$

This hybrid approach ensures that the final attribution parameter $\alpha$ falls within a range from the calculated value based on an objective method. If the subjectively specified value is too small or too big, the final result will be retained at the lower or upper bound. In so doing, the level of subjectivity in finding an appropriate value of $\alpha$ is effectively contained.

\section{Conclusion}

By analyzing existing methods (Wang and Lei et al. 2007) to operationalize the $D_{\alpha}$ operator proposed by Atanassov (1989, 1995, 
2008), this paper first proposes an improved formula to determine the key parameter $\alpha$ in characterizing hesitations in IFN assessments. Comparative studies reveal the key properties of the proposed method. Then a hybrid framework is put forward to integrate a DM's subjective judgment into the proposed objective approach in finding an appropriate value of $\alpha$. This further characterization of hesitations in IFN assessments is especially useful when the hesitation levels are high as the traditional score-function-based ranking approach tends to omit hesitations. By properly attributing hesitations to membership and nonmembership functions, one can obtain more reliable and confident decision results.

\section{Acknowledgment}

The authors are grateful for the two anonymous referees for their constructive comments that have helped to improve the quality of this paper. Minji Huang is grateful for the financial support from the Fumin Foundation so that she could work on this joint research while visiting the Odette School of Business at the University of Windsor in Canada between February and July 2011. Kevin W. Li would like to acknowledge the financial support from the Natural Sciences and Engineering Research Council of Canada (NSERC) under its Discovery Grant program and the Natural Science Foundation of China (Nos. 71272129 and 71271188). Kevin W. Li also wishes to express his gratitude to the Japan Society for the Promotion of Science (JSPS) for its Invitation Fellowship that allowed him to work on the research reported in this article during his sabbatical leave between 20 June and 19
December 2011 in the Department of Value and Decision Science at the Tokyo Institute of Technology in Japan.

\section{References}

[1] Atanassov, K.T. (1986). Intuitionistic fuzzy sets. Fuzzy Sets and Systems, 20(1): 87-96

[2] Atanassov, K.T. (1989). More on intuitionistic fuzzy sets. Fuzzy Sets and Systems, 33(1): 37-45

[3] Atanassov, K.T. (1995). Remarks on the intuitionistic fuzzy sets-III. Fuzzy Sets and Systems. 75(3): 401- 402

[4] Atanassov, K.T. (2008). My personal view on intuitionistic fuzzy sets theory. Studies in Fuzziness and Soft Computing, 220: 23-43

[5] Atanassova, V. (2006). Strategies for decision making in the conditions of intuitionistic fuzziness. Advances in Soft Computing, 33: 263-269

[6] Boran, F.E., Genc, S., Kurt, M., \& Akay, D. (2009). A multi-criteria intuitionistic fuzzy group decision making for supplier selection with TOPSIS method. Expert Systems with Applications, 36: 11363-11368

[7] Chen, S.M., \& Tan, J.M. (1994). Handling multicriteria fuzzy decision-making problems based on vague set theory. Fuzzy Sets and Systems, 67(2): 163-172

[8] Chen, T.Y. (2011). A comparative analysis of score functions for multiple criteria decision making in intuitionistic fuzzy settings. Information Sciences, 181(17): 3652-3676

[9] Chen, T.Y., Wang, H.P., \& Lu, Y.Y. (2011). A multicriteria group decision-making approach based on interval-valued intuitionistic fuzzy sets: A comparative 
perspective. Expert Systems with Applications, 38(6): 7647-7658

[10]Chen, Z.P., \& Yang, W. (2012). A new multiple criteria decision making method based on intuitionistic fuzzy information. Expert Systems with Applications, 39: 4328-4334

[11]Herrera, F., Marttínez, L., \& Sánchez, P.J. (2005). Managing non-homogeneous information in group decision making. European Journal of Operational Research , 166 (1): 115-132

[12]Hong, D.H. \& Choi, C.H. (2000). Multicriteria fuzzy decision-making problems based on vague set theory. Fuzzy Sets and Systems, 114(1): 103-113

[13]Li, D.F. (2002). Fuzzy multi-objective many-person decision makings and games. National Defense Industry Press, pp. 88-93. (In Chinese)

[14]Li, K.W., Karray, F., Hipel, K.W., \& Kilgour, D.M. (2001). Fuzzy approaches to the game of chicken. IEEE Tranactions on Fuzzy Systems, 9(4): 608-623

[15]Li, K.W. \& Wang, Z.J. (2010). Notes on "Multicriteria fuzzy decision-making method based on a noval accuracy function under interval-valued intuitionistic fuzzy environment", Journal of Systems Science and Systems Engineering, 19(4): 504-508

[16]Liu, H.W., \& Wang, G.J. (2007). Multi-criteria decision making methods based on intuitionistic fuzzy sets. European Journal of Operational Research, 179: 220-233

[17] Tan, C., \& Chen, X. (2010). Intuitionistic fuzzy Choquet integral operator for multi-criteria decision making. Expert
Systems with Applications, 37: 149-157

[18]Wang, Y., Lei, Y. J., \& Wang, J. (2007). The conversion method from IFS to ZFS base on difference correcting. Aeronautical Computing Technique, 3: 78-11. (In Chinese)

[19]Wang, Z-J, \& Li, K.W. (2012). An interval-valued intuitionistic fuzzy multiattribute group decision making framework with incomplete preference over alternatives. Expert Systems with Applications, 39(18): 13509-13516

[20]Wang, Z.J., Li, K.W, \& Wang, W.Z. (2009). An approach to multiattibute decision making with interval-valued intuitionistic fuzzy assessments and imcomplete weights. Information Sciences, 179: 3026-3040

[21]Wang, Z.J., Li, K.W., \& Xu, J.H.( 2011). A mathematical programming approach to multi-attribute decision making with interval-valued intuitionistic fuzzy assessment information. Expert Systems With Applications, 38(10): 12462-12469

[22]Wang, Z.J., \& Qian, E. Y. (2007). A vague-set-based fuzzy multi-objective decision making model for bidding purchase. Journal of Zhejiang University(Science A), 8(4): 644-650

[23]Xu, Z.S. (2011). Approaches to multiple attribute group decision making based on intuitionistic fuzzy power aggregation operators. Knowledge-Based Systems, 24(6): 749-760

[24]Xu, Z.S., \& Chen, Q. (2011). A multi-criteria decision making procedure based on interval-valued intuitionistic fuzzy Bonferroni means. Journal of Systems Science and Systems Engineering, 20(2): 
217-228

[25]Xu, Z.S., \& Yager, R.R. (2008). Dynamic intuitionistic fuzzy multi-attribute making. International Journal of Approximate Reasoning, 48: 246-262

[26]Zadeh, L.A. (1965). Fuzzy sets. Information and Control, $8(3): 338-353$

Minji Huang is a Ph.D. candidate in Control Theory and Engineering at Xiamen University, Fujian, China. She received her B.A.Sc. in System Engineering from Xiamen University in 1996, and M.A.Sc. in Computer Science \& Technology from Fuzhou University in 2003. She spent six months as a visiting student scholar in the Odette School of Business at the University of Windsor in Canada in 2011. She is a Senior Engineer in Fujian Educational Examinations Authority, China. Her project entitled "The Strategic Research for The Future Development of National Computer Rank Examination" was awarded a third-class science and technology progress prize by Xiamen City in 2002. She also won an Outstanding Master Thesis Award from Fuzhou University, China, in 2003. Her current research interest focuses on Intelligent Decision Support Systems Design.
Kevin W. Li received his B.Sc. degree in control sciences and M.A.Sc. degree in systems engineering from Xiamen University, Xiamen, China, in 1991 and 1994, respectively, and Ph.D. degree in systems design engineering from the University of Waterloo, Waterloo, Canada in 2003. Dr. Li is an associate professor in the Odette School of Business at the University of Windsor, Windsor, Canada. His research interests are concerned with finding equitable and sustainable solutions to complex decision problems. His papers have been published in reputable journals such as Computers and Operations Research, European Journal of Operational Research, IEEE Transactions on Fuzzy Systems, IEEE Transactions on Systems, Man, and Cybernetics, Information Sciences, International Journal of Production Economics, Journal of the Operational Research Society, and Water Resources Research. During June and December 2011, Dr. Li visited the Tokyo Institute of Technology as a Japan Society for the Promotion of Science (JSPS) Fellow. His research has been supported by two Natural Sciences and Engineering Research Council of Canada (NSERC) Discovery Grants and several internal grants from the University of Windsor. 